\title{
ARQUITECTURA HARDWARE/SOFTWARE PARA UN PROTOTIPO DE POZO INTELIGENTE EN UN CAMPO PETROLERO MADURO
}

\section{HARDWARE / SOFTWARE ARCHITECTURE FOR AN INTELLIGENT WELL PROTOTYPE BASED ON MECHANICAL PUMPING}

\author{
MEng. Jorge Enrique Meneses Flórez ${ }^{*}$, MSc. Diana Paola Meneses Salazar ** \\ * Universidad Industrial de Santander (UIS), Escuela de Ingeniería Mecánica. \\ Grupo de Investigación en Conectividad y Procesado de Señales - CPS \\ Ciudad Universitaria, Bucaramanga, Santander, Colombia. \\ Tel.: (+577) 6344000 Ext. 2483/2829. \\ E-mail: jmeneses@uis.edu.co \\ **Universidad de los Andes, Grupo de Mecánica Computacional. \\ E-mail: dp.meneses60@uniandes.edu.co.
}

Resumen: Se presenta la arquitectura del SmartOMP, producto mecatrónico diseñado y construido como una solución propia dirigida a un campo petrolero maduro, a través del cual se puede identificar con precisión y rapidez las fallas en un pozo, con el fin de disminuir el riesgo operacional y mejorar la producción, incorporando un sistema automático inteligente que reconoce los patrones de falla del sistema de bombeo mecánico. SmartOMP obtiene en tiempo real el dinagrama de fondo de pozo, utilizando dos sistemas interconectados de manera inalámbrica; un sistema se ubica en cabeza de pozo sobre la barra pulida para obtener de manera permanente, las señales instantáneas de aceleración y carga; el otro sistema, ubicado a distancia, recibe los datos de carga y aceleración, procesándolos para obtener en primera instancia el dinagrama de cabeza de pozo, y mediante procesamiento computacional, obtener el dinagrama de fondo de pozo.

Palabras clave: Dinagrama, bombeo mecánico, campo maduro, ecuación de onda,

\begin{abstract}
The architecture of SmartOMP is presented, a mechatronic product designed and built as a proprietary solution aimed at a mature oil field, through which failures in a well can be accurately and quickly identified, in order to reduce operational risk and improve production, incorporating an intelligent automatic system that recognizes the failure patterns of the mechanical pumping system. SmartOMP obtains the bottomhole dynagram in real time, using two wirelessly interconnected systems; a system is located at the wellhead on the polished bar to obtain permanent, instantaneous acceleration and load signals; The other system, located at a distance, receives the load and acceleration data, processing them to obtain the wellhead dynagram in the first instance, and by computational processing, obtain the well bottom dynagram.
\end{abstract}

Keywords: Dynagram, mechanical pumping, mature field, wave equation 


\section{INTRODUCCIÓN}

\subsection{Campo maduro y Pozo inteligente}

Un campo petrolero "maduro" es aquel donde se ha rebasado el índice de producción previsto en el esquema original de explotación y aunque la producción se encuentra en declinación, ella es rentable. Los costos de producción podrían convertirlo en un campo "marginal", y su producción no ser rentable. Ante la escasez de petróleo y en las épocas de precio alto del crudo, los campos maduros adquieren importancia para los países que los poseen, siendo estos una forma de incrementar la producción.
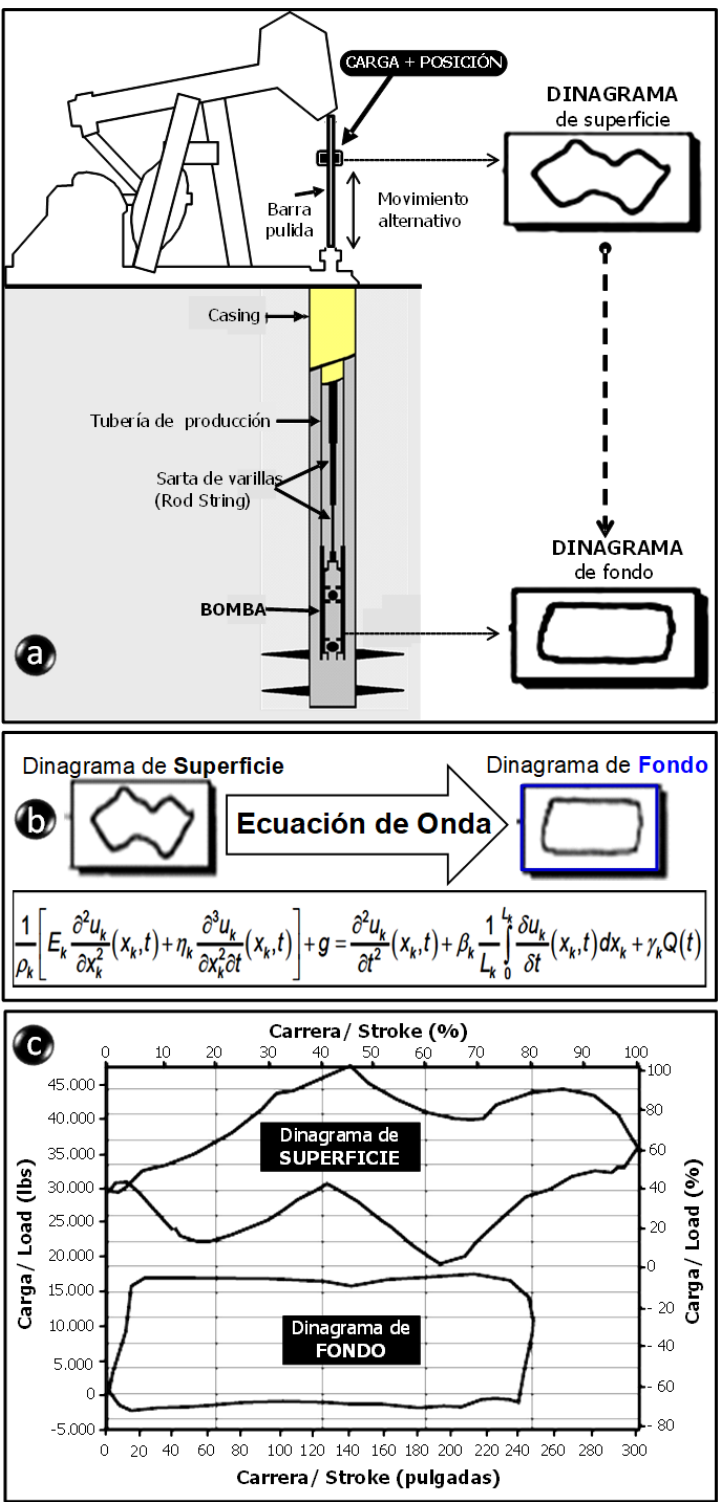

Fig. 1. Sistema de bombeo mecánico y dinagramas

Universidad de Pamplona I. I. D. T. A.
Los pozos petroleros son activos cuyo valor decrece de forma permanente, en consecuencia, los ingenieros de producción están constantemente buscando formas de optimizar la producción de los mismos, con el mínimo desplazamiento a los sitios. Campos y pozos inteligentes (Intelligent wells Smart Fields), son conceptos modernos que conducen al aumento de la producción (Sletcha et al., 2020; de Best, 2012; de Best, 2006) y que abarcan todas aquellas soluciones centradas en las tecnologías de información y de automatización (Pari y Kabir, 2009; Gao, 2007), que permiten aumentar la productividad permitiendo una reacción rápida y oportuna ante acontecimientos inesperados durante la vida de un yacimiento, disminuyendo la necesidad de intervención humana, lo cual conduce a crear valor mediante la reducción de los costos de intervención, reduciendo por ende los costos operativos (Al-Mubarak, 2008). Ante tantas bondades se ha generado un interés creciente en la industria petrolera de poseer pozos y campos inteligentes (Moises et al., 2008).

En Colombia, nuestra industria petrolera se ha dedicado a consumir los componentes hardware y software proporcionados por empresas extranjeras, componentes que son cerrados (propietarios) generando con ello una absoluta dependencia. El costo asociado a la adquisición de este tipo de tecnologías de punta, hace poco atractiva su utilización en campos maduros, dado que el nivel de producción, algunas veces, no permite un margen de utilidad que justifique su compra. La (necesidad) oportunidad radica en el diseño, construcción e implantación de herramientas hardware y software de bajo costo, potentes y robustas, que contribuyan al desarrollo propio del concepto de pozo y campo inteligente.

\subsection{Campo Escuela Colorado y proyecto 8556}

En 2006, nace el Campo Escuela Colorado (CEC) bajo la firma de un convenio interadministrativo entre la UIS y ECOPETROL, por medio del cual la compañía estatal petrolera hizo entrega a la UIS, por 10 años, del Campo Colorado. CEC tuvo como visión el promover la investigación científica y el desarrollo tecnológico de la cadena productiva de los hidrocarburos, para lograr su producción sostenible y su articulación al desarrollo económico y social del país. Campo Colorado es un campo maduro que se encuentra localizado en la vereda Los Colorados, corregimiento de Yarima, municipio de San Vicente de Chucurí (Santander). El campo posee acumulaciones de aceite liviano y gas con gravedad API 36 a 42 y utiliza para el 
levantamiento artificial del crudo, sistemas de bombeo mecánico.

En el marco de CEC y en correspondencia con su visión, se desarrolló el proyecto de investigación "8556 Prototipo de un pozo inteligente para CEC", financiado por la Vicerrectoría de Investigación y Extensión de la UIS. En el proyecto se diseñó y desarrolló una solución de hardware y software, para establecer las bases propias para el concepto de pozo y campo inteligente, a través de la cual se pudiera identificar con precisión y rapidez los problemas (fallas) en un pozo, con el fin de disminuir el riesgo operacional y mejorar la producción, incorporando un sistema automático que reconozca los patrones de falla del sistema de bombeo mecánico, a partir de los dinagramas de fondo, anticipando los problemas a través de su pronta identificación. En el presente artículo se describe la arquitectura hardware/software diseñada y desarrollada.

\subsection{Bombeo Mecánico y Dinagramas}

Cuando la energía de un pozo de petróleo es insuficiente para llevar el crudo a la superficie por sí mismo, se recurre a un Sistema de Levantamiento Artificial (SLA), mecanismo por el cual se levanta el crudo desde la formación a una determinada tasa. El principal SLA en el mundo es el denominado "bombeo mecánico", se estima que el $90 \%$ de los pozos con SLA lo utilizan (Gabor, 2003). El bombeo mecánico (Fig. 1) utiliza una bomba de desplazamiento positivo ubicada en el fondo del pozo (a miles de metros) que recibe un movimiento lineal alternativo generado por una unidad de bombeo situada en la superficie. La transmisión del movimiento a la bomba se hace mediante la unión de una serie de varillas (sarta) de bombeo. La bomba es el núcleo del sistema de bombeo, y su desempeño tiene un impacto directo en el nivel de beneficio económico del pozo y del yacimiento de petróleo (Sanchez et al., 2007). Es evidente la dificultad para realizar mediciones directamente sobre la bomba, en el fondo del pozo. Para monitorear el desempeño del sistema de bombeo, un dispositivo denominado dinamómetro de superficie, se une a la barra pulida en la cabeza del pozo, el cual mide la fuerza (carga) en función de su posición, para generar una curva que suele denominarse dinagrama de superficie (McCoy y Podio, 1995). El dinagrama es una curva cerrada, en la cual el eje $\mathrm{x}$ representa el desplazamiento de la sarta de varillas en cabeza de pozo, y el eje y representa la carga (fuerza) presente para dicha posición, describiendo la relación carga- desplazamiento en un ciclo de bombeo. Modelando el comportamiento del crudo bombeado, y el comportamiento de la sarta de varillas que conecta la superficie con el fondo del pozo, se obtiene una ecuación diferencial de segundo orden en derivadas parciales y sus correspondientes condiciones de frontera, denominada "Ecuación de Onda", y aplicando procesos matemáticos se obtiene, a partir del dinagrama de superficie, el dinagrama de fondo (Fig. 1), el cual se constituye en la fuente de información fundamental para diagnosticar cualquier falla del sistema de bombeo mecánico.

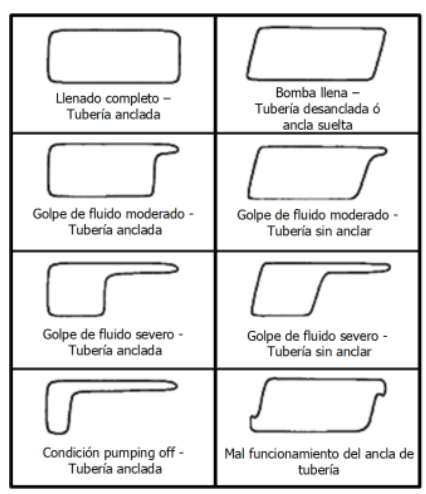

Fig. 2. Formas típicas de DINAGRAMAS de fondo

Como se puede inferir, los dinagramas de fondo son la clave para determinar el funcionamiento de un sistema de bombeo mecánico, ya que mostrarán formas disímiles que podrían corresponder a una operación normal o a situaciones de fallas (Fig. 2). Normalmente el diagnóstico de las fallas del sistema de bombeo, se ha basado en la interpretación visual de los dinagramas por parte de un ingeniero experto (Dickinson y Jennings, 1990), lo que conlleva a que se vea afectado por factores tales como: imposibilidad de diagnosticar en tiempo real, la complejidad del sistema de bombeo mecánico, la diversidad de formas de los dinagramas, la experiencia y la habilidad del ingeniero que diagnostica. En conclusión, la arquitectura de requerida para que el sistema sea "automático", implica no solamente capturar y procesar las variables físicas que permitan obtener los dinagramas de superficie y de fondo, sino que adicionalmente requiere de un nivel software que permita prescindir del experto humano y diagnosticar las fallas de manera automática. 


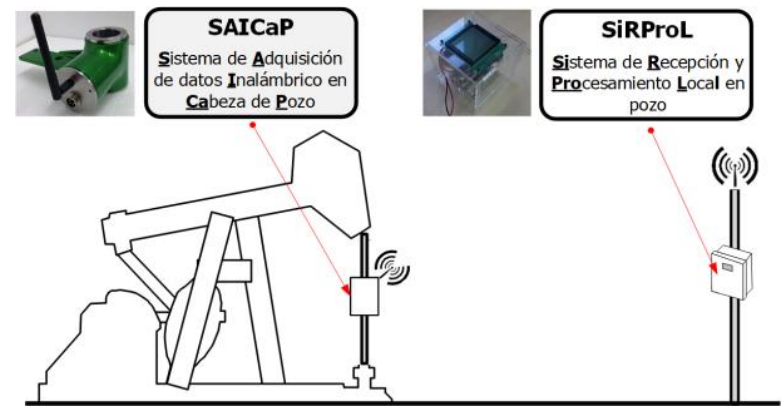

Fig. 3. SmartOMP: los dos sistemas

\section{ARQUITECTURA}

Se diseñó y construyó SmartOMP (Smart Oil Mechanical Pumping), un producto de naturaleza mecatrónica (hardware/software) para la obtención automática, en tiempo real, del dinagrama de fondo de pozo y el diagnóstico del sistema de bombeo. SmartOMP fue concebido para ser instalado en el pozo 74 de CEC y posee dos sistemas conectados entre sí de manera inalámbrica (Fig. 3):

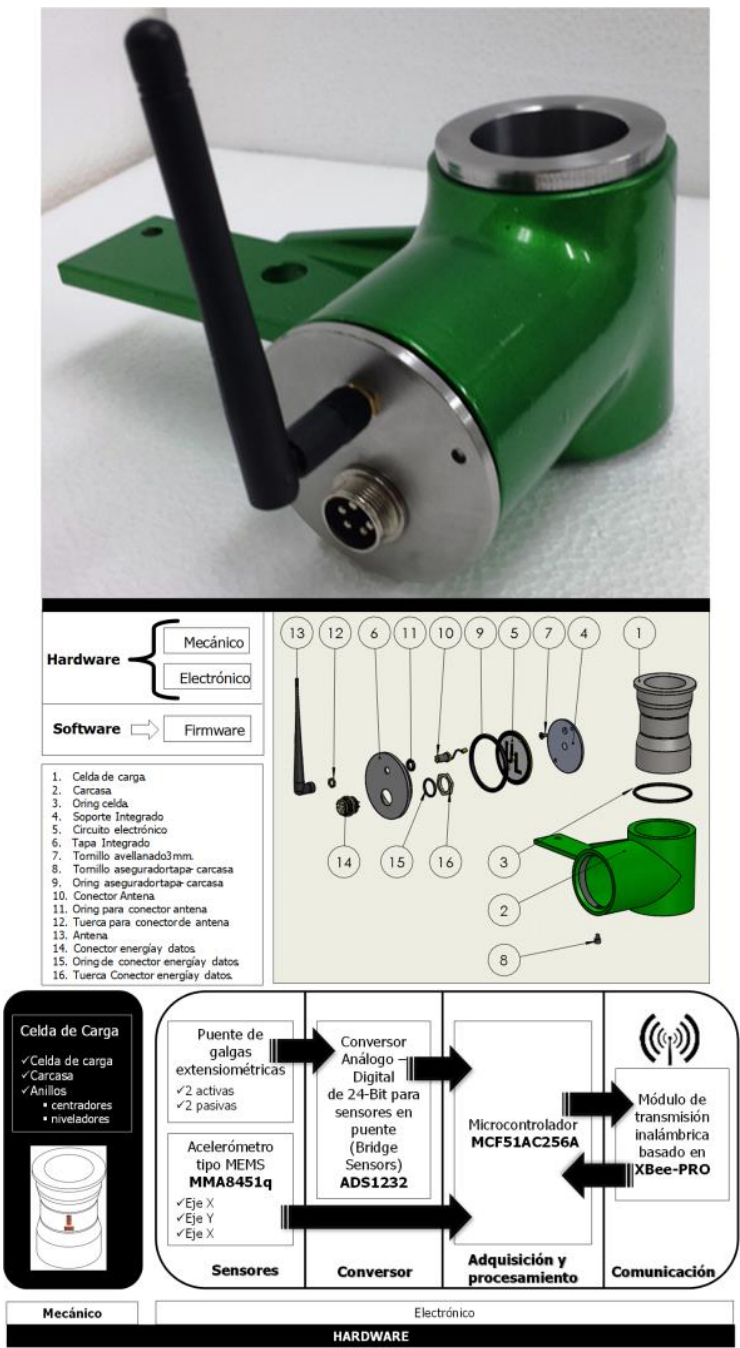

Fig. 4. SAICaP, ensamble y estructura hardware.

i. El Sistema de Adquisición de datos Inalámbrico en Cabeza de Pozo, de ahora en adelante SAICaP, ubicado en cabeza de pozo sobre la barra pulida, obtiene en tiempo real y de manera permanente, las señales instantáneas de aceleración y carga presentes en la barra de pulida de la unidad de levantamiento artificial. Obtenidas y procesadas las señales mediante hardware y software, éstas son transmitidas vía inalámbrica (ZigBee) a otro sistema remoto que se encarga de obtener los dinagramas de fondo.

ii. El Sistema de Recepción y Procesamiento Local en pozo, de ahora en adelante SiRProL, recibe vía inalámbrica los datos de carga y aceleración adquiridos por SAICaP, procesándolos para obtener en primera instancia el dinagrama de superficie, y mediante procesamiento computacional, obtener el 
dinagrama de fondo, para su posterior diagnostico automático mediante una herramienta software desarrollada, basada en inteligencia artificial, utilizando algoritmos NEURO Fuzzy.

\subsection{SAICaP}

El sistema SAICaP desarrollado (Fig. 4) es un sensor inteligente de naturaleza embebida, basado en el $\mu$ controlador MCF51AC256 que se encarga de realizar las tareas de muestreo, pre procesamiento y almacenamiento de las variables físicas de aceleración y carga. Su estructura está constituida por un nivel hardware con componentes mecánicos y electrónicos, y por un nivel software (firmware).

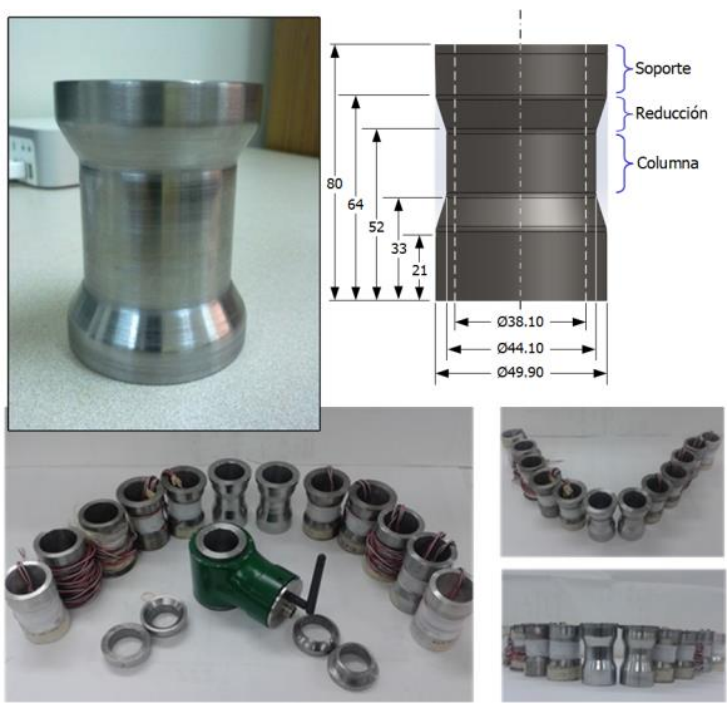

Fig. 5. Celdas de carga y anillos

\subsubsection{Hardware mecánico - Celda de carga}

Tiene como función medir directamente la carga en la barra pulida y contener todos los dispositivos electrónicos del SAICaP. Se diseñó y manufacturó una celda de carga de 10.000 lb a compresión, de fijación permanente y resistente a las condiciones ambientales presentes en un pozo petrolero; su diseño básico consiste un cilindro hueco que se adapta fácilmente al montaje en la unidad de bombeo, e incluye un sistema de centrado y auto nivelación. Se desarrolló una carcasa totalmente hermética para albergar tanto la celda de carga como los circuitos electrónicos de sensado y transmisión de datos, preparada para una certificación anti-explosión y la respectiva norma de protección IP (Fig. 5).

\subsubsection{Hardware electrónico del SAICaP}

El hardware electrónico (Fig. 6) se diseñó teniendo como cerebro un $\mu$ controlador de 32 bits, (MCF51AC256) y realiza las siguientes tareas: a) capturar la carga instantánea del pozo, convirtiendo, través del uso de un puente de Wheatstone, la deformación mecánica de las galgas extensiométricas, en una señal eléctrica digital; b) capturar la aceleración, proveniente del movimiento alternativo de la barra pulida del pozo; c) procesar las señales de aceleración y carga; d) establecer comunicación con el SiRProL. El hardware electrónico del SAICaP está basado en los siguientes componentes: Puente de galgas extensiométricas, Acelerómetro tipo MEMS (MMA8451Q), Conversor A/D (ADS1232) de 24Bit para sensores en puente, $\mu$ controlador (MCF51AC256A) de Freescale, XBEE PRO. Como alternativa al Acelerómetro tipo MEMS (MMA8451Q), se exploró la posibilidad de obtener la posición instantánea de la barra pulida mediante video (Meneses et al., 2017), desarrollando un proyecto paralelo.

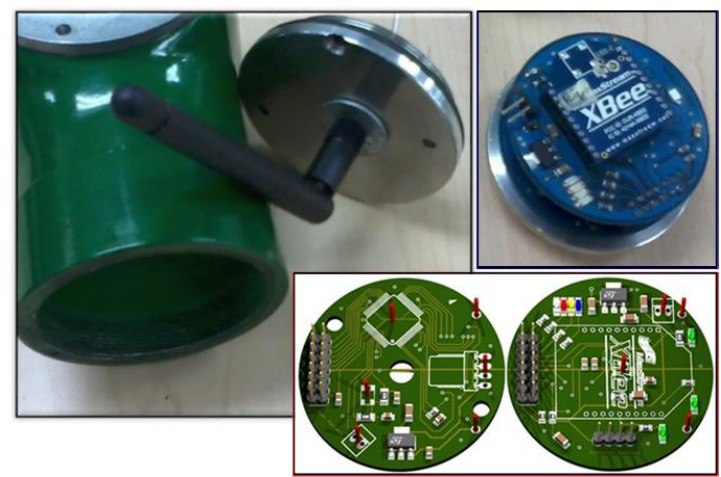

Fig. 6. Prototipo final del hardware electrónico del SAICaP

Galgas extensiométricas: Dado que el objetivo era diseñar y construir un sensor de carga (celda), o sea un instrumento de medición, el tipo de aplicación fue de "galgas para transductor", siendo estas especiales y de alta gama. Como proveedor se seleccionó a Micro-Measurements de Vishay y se requirió de la fabricación especial de las galgas. En la Figura 7 se muestran galgas seleccionadas para este proyecto, cuyas características son:

a) galgas para transductor tipo columna, con resistencias de 1000 y $350 \Omega$. Bidireccionales dobles a $90^{\circ}$, para un puente de Wheatstone de cuatro galgas, en el cual dos son activas (en 
dirección a la carga) y dos son pasivas (perpendiculares a la línea de acción de la carga).

b) La serie de la galga determina la combinación entre el material del hilo metálico y el material de respaldo, se seleccionó la combinación de N2A (aleación de constantan) y N2K (aleación de Karma) ambas sobre un soporte de película de poliimida laminado, con la rejilla recubierta por una película delgada de polímero $(0.013 \mathrm{~mm})$, y tabs para soldar descubiertos.

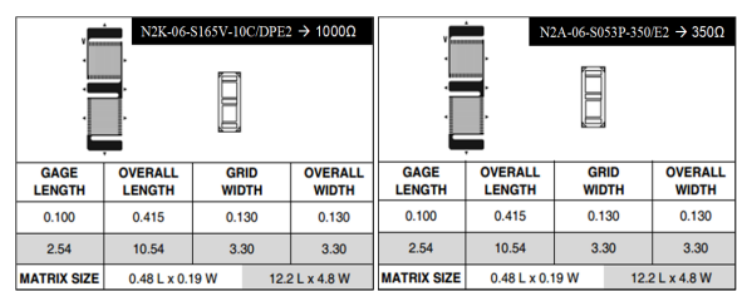

Fig. 7. Galgas extensiométricas seleccionadas

Acelerómetro tipo MEMS (MMA8451Q): Para capturar la aceleración instantánea de la barra pulida, fue seleccionado el MMA8451Q, que es un acelerómetro inteligente (smart) tipo MEMS de tres ejes (Meneses et al., 2015; Gómez et al., 2013), con principio capacitivo, 14 bits de resolución y bajo consumo de energía. Su selección se basó en que: a) Posee filtros de datos pasa bajos (low-pass) y pasa altos (high-pass), lo que facilita el análisis de los datos en los eventos de sacudida y transiciones más rápidas; b) posee funciones integradas con opciones flexibles programables por el usuario, mediante la configuración de dos pines de interrupción; c) Posee funciones de interrupción embebidas, que permiten el ahorro de energía evitando que el $\mu$ controlador procese de forma continua los datos; d) el acelerómetro puede ser configurado para generar señales de interrupción de activación inerciales, mediante las cuales puede supervisar los acontecimientos y permanecer en un modo de bajo consumo durante los períodos de inactividad.

Conversor A/D ADS1232 (24-Bit Bridge Sensors): La fuerza instantánea (carga compresiva) actuante sobre la celda de carga, es captada mediante el puente de Wheatstone que entrega una señal con variaciones en el orden de los $\mu \mathrm{V}$. La señal captada se convierte a digital, mediante el ADS1232 de tecnología $\sum-\Delta$. El ADS1232 es un conversor analógico/digital de alta resolución (24 bits), posee de forma embebida un amplificador de ganancia programable (PGA), bajo nivel de ruido y un oscilador interno. El ADS1232 proporciona una solución final para aplicaciones de sensado que utilicen puentes (bridge), entre ellos para sensores de carga compresivas basadas en galgas extensiométricas, razón de su selección para el desarrollo de este proyecto. La arquitectura $\sum-\Delta$ utiliza técnicas de procesamiento digital para adquirir y procesar las señales analógicas y presentaba la mayor ventaja para ser utilizada por su precisión, teniendo en cuenta que se requería adquirir una señal de muy baja tensión.

Microcontrolador MCF51AC256: Es el encargado de adquirir las señales provenientes del sensor de aceleración y del conversor ADS1232; el acelerómetro utiliza el protocolo $\mathrm{I}^{2} \mathrm{C}$ (InterIntegrated Circuit) mientras que el conversor $\mathrm{A} / \mathrm{D}$ utiliza el protocolo "Serial SCI", el cual es una variante del protocolo SCI; estos protocolos de comunicación digital permiten enviar y recibir datos al $\mu$ controlador, tales como interrupciones de los dispositivos o incluso datos de calibración entre los dispositivos. El $\mu$ controlador puede alcanzar velocidades de procesamiento de hasta $25 \mathrm{MHz}, \mathrm{y}$ cuenta con un módulo especial llamado RTC que tiene la capacidad de generar interrupciones periódicas, las cuales fueron utilizadas para el muestreo de los datos.

XBEE PRO: Los módulos Xbee Pro ofrecen un alcance en línea de vista directa de hasta $1 \mathrm{~km}$, son de fácil adquisición y ofrecen la versatilidad de incorporarse e implementar diferentes tipos de redes, lo que lo hacía ideal para su aplicación en proyecto desarrollado. La transmisión inalámbrica entre los dos sistemas (SAICaP y SiRProL) se realiza a una frecuencia de $2,4 \mathrm{GHz}$, utilizando el protocolo Zigbee a través de los módulos XBEE Pro, que se comunican con el microcontrolador mediante el protocolo serial SCI.

\subsubsection{Firmware del SAICaP}

El firmware fue desarrollado sobre Codewarrior V6.3. El SAICaP, desde el momento de su energización, permanece en forma cíclica realizando la captura y el pre-procesamiento (adquisición) de las señales de aceleración y carga, llenando un buffer en el cual se pueden almacenar 700 datos. La interrupción SCI permite saber el momento preciso en el cual el "buffer de datos" recibe un carácter proveniente del sistema SiRProL solicitando el envió de los datos. Si el buffer de datos está lleno y el SAICaP durante 120 segundos no recibe petición por parte del sistema SiRProL, desocupa el buffer y comienza un nuevo ciclo de captura de señales y llenado del buffer (Fig. 8). 


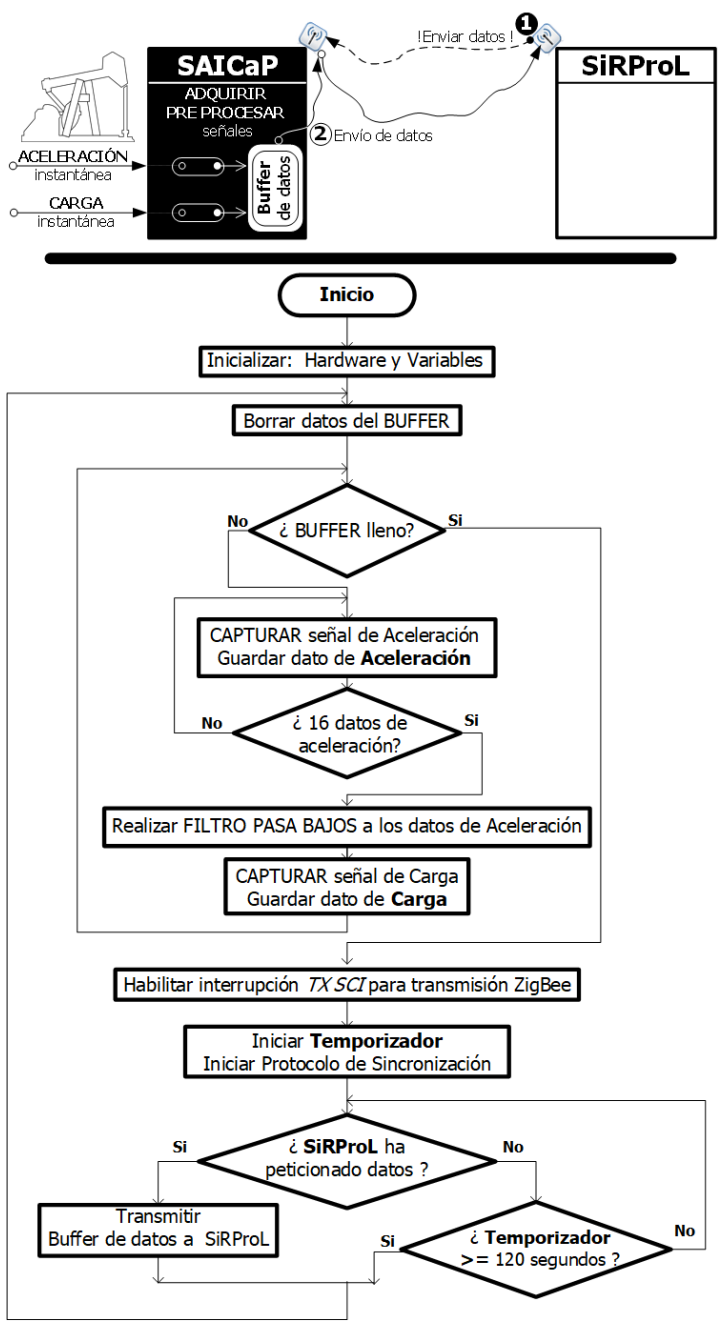

Fig. 8. Firmware del SAICaP

Los módulos utilizados para que el SAICaP funcione como un sensor inalámbrico fueron:

- ${ }^{2} \mathrm{C}$ : realiza la comunicación con el acelerómetro MMA8451Q, y puede establecer comunicación con otros dispositivos que posean $\mathrm{I}^{2} \mathrm{C}$.

- Serial-SCI: utiliza una señal para reconocer cuando un dispositivo esclavo está listo para enviar alguna señal, o en este caso, ha terminado una conversión y el dato está listo para ser leído.

- SCI: Este bus es utilizado para la comunicación con el módulo XBEE, siendo el encargado de realizar el puente de comunicación inalámbrico por medio del protocolo Zig-Bee.

- RTC: Es utilizado para establecer los tiempos del muestreo de los datos por medio de una interrupción en el procesador.
Este sistema se ubica cerca al pozo petrolero (20 a 50 metros), es el cerebro del producto mecatrónico desarrollado (SmartOMP). La concepción de diseño se fundamentó en desarrollarlo como un sistema embebido alrededor de una tarjeta madre comercial, a través de la cual se pudiera desarrollar toda la potencia de cálculo que se requería, para procesar en tiempo real y de manera local, o sea en el pozo, las señales de aceleración y carga, con el fin de obtener el dinagrama de fondo de pozo y diagnosticar el sistema, ver Figuras 3 y 9 .

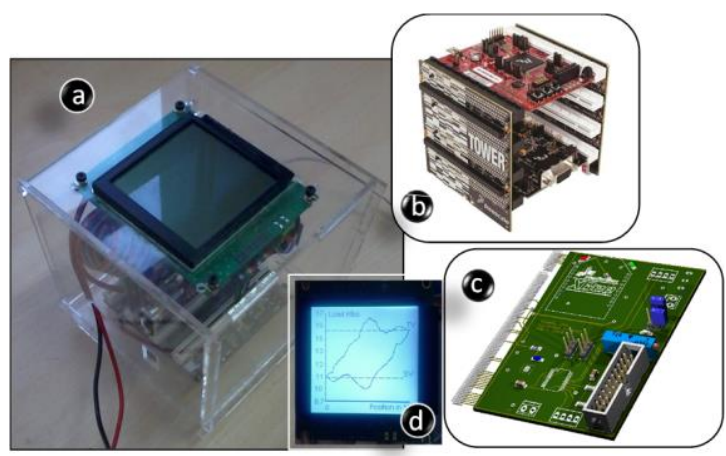

Fig. 9. Prototipo final del SiRProL

\subsubsection{Hardware del SiRProL}

El prototipo final del SiRProL (Fig. 9a) se diseñó y desarrolló alrededor de una tarjeta madre TWRK70F120M de Freescale (Fig. 9b), y su estructura hardware se observa en la Figura 10. La tarjeta incorpora la MCU ( $\mu$ controlador) Kinetis K70 mediante la cual se procesan los datos. El sistema Tower permite desarrollar e incorporar plataformas hardware propias para aplicaciones específicas, utilizando sus conectores y módulos elevadores.

Para dotar a SiRProL de las funciones requeridas, se diseñó y fabricó una PCB (Printed Circuit Board) que se pudiera integrar al sistema Tower, mediante el uso de un módulo elevador (Fig. 9c) y conexión a la tarjeta madre, a través de su conector primario. (Araque, JA, Rodríguez, JLD, Guerrero A. 2017). (Bermeo, WL, Jr, AB de Souza, Fernández T, Honorio D, Nogueira dos Reis L, Barreto L. 2017).

\subsection{SiRProL}




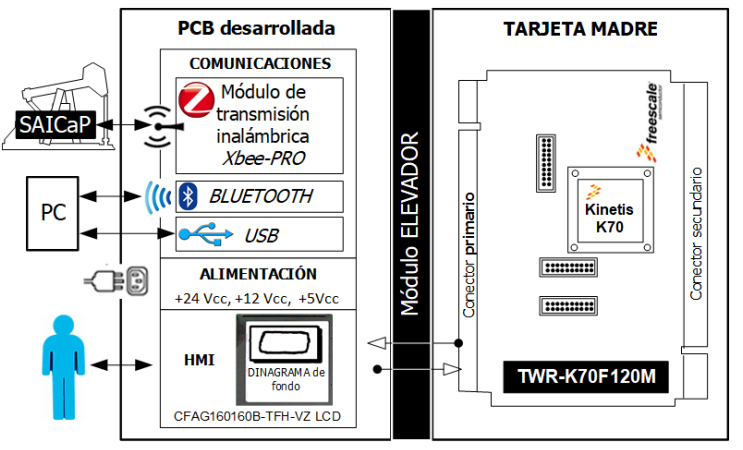

Fig. 10. Componentes hardware del SiRProL

Tarjeta madre $\rightarrow$ TWRK70F120M: es una tarjeta de desarrollo para las MCU Kinetics K70, que utiliza $\mu$ controladores ARM ${ }^{\circledR}$ Cortex ${ }^{\mathrm{TM}}-\mathrm{M} 4$ de 32 bits, los cuales poseen bajo consumo de potencia, gran escalabilidad e integran múltiples funciones. Incorpora una unidad especial de punto flotante (FPU), la cual funciona de manera similar a una Unidad Lógico Aritmética (ALU. Además, su $\mu$ controlador posee un reloj de tiempo real que permite llevar la cuenta del tiempo a la vez que se ejecuta el programa. Incluye también: Un (1) MB de memoria flash, controlador LCD gráfico, IEEE® 1588 Ethernet, USB 2.0 completo y de alta velocidad, encriptación vía hardware, controlador de memoria flash NAND.

$P C B$ desarrollada: permite integrar los dispositivos electrónicos externos: la pantalla LCD, el modem Bluetooth, el módulo XBEE, la interfaz USB para comunicación con el computador, y la alimentación para manejar $+24 \mathrm{Vcc} /+12 \mathrm{Vcc} /+5 \mathrm{Vcc}$

XBEE PRO: $\mathrm{Al}$ igual que para el sistema SAICaP, se incluye un módulo XBEE PRO que permite la comunicación inalámbrica entre ambos sistemas.

Bluetooth ${ }^{\circledR}$ modem: Se incorporó el módulo Bluetooth inalámbrico "BlueSMiRF Silver" con antena integrada y comunicación serial, el cual permite realizar una comunicación Bluetooth para transmitir los datos que han sido procesados, hacia otros dispositivos externos tales como: un PC, un IPAD, un Smartphone, etc

Alimentación: El prototipo final cuenta con un regulador de tensión de 24 a 12 Volts de Texas instruments, con tecnología LDO.

Conversor USB-RS232: basado en un chip controlador FTDI, permite la comunicación mediante cable USB con un PC, simulando un puerto serial por el cual se realiza la comunicación mediante caracteres de 8 bits.

HMI Crystalfontz CFAG160160B-TFH - VZ LCD: Para la visualización del dinagrama de fondo y de los diagnósticos al sistema de bombeo, se utilizó una LCD, monocromática de 160x160 pixeles, comunicación paralela de 8 bits, memoria SRAM de $4 \mathrm{~Kb}$ y un controlador grafico LC7981(Fig. 9d).

\subsubsection{Software (Firmware) del SiRProL}

El software (firmware) fue desarrollado sobre el compilador Codewarrior V6.3 para $\mu$ controladores de la empresa Freescale. SiRProL lanza una petición de "envío de datos" al sistema SAICaP y de existir datos en su buffer, se sincroniza y recibe los datos (Fig. 3 y 8a). Teniendo los datos de aceleración y carga instantánea, realiza los procesamientos necesarios para tratar los datos y obtener en primera instancia el dinagrama de superficie y posteriormente el dinagrama de fondo y realizar un diagnóstico del estado del sistema.

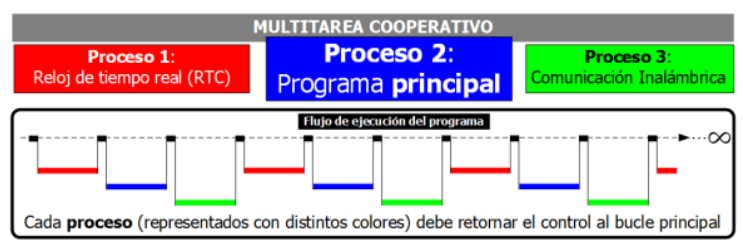

Fig. 11. Procesos software del SiRProL

El software desarrollado para el SiRProL se encuentra basado en concepto general de segmentación electrónica para procesadores (Pipelining), el cual permite realizar múltiples tareas de manera óptima, mejorando el rendimiento en aspectos como la latencia y por consiguiente los tiempos de procesamiento. Consiste en tres procesos corriendo de manera independiente (software multitarea), y mediante el uso de interrupciones se priorizan las tareas más críticas, (Fig. 11). El proceso uno se encarga de la tarea principal, la cual consiste en mantener un reloj de tiempo real (RTC), de manera que se pueda obtener la hora precisa de obtención y procesamiento de los datos. El proceso dos es el "núcleo o programa principal", y se encarga de desarrollar las tareas específicas de: comunicación inalámbrica, procesamiento de los datos, y la organización en general de la ejecución de las tareas. El proceso tres se encarga de las comunicaciones, para establecer conexiones con el SAICAP o con una interfaz de usuario en un computador personal (PC) por medio de Bluetooth o conexión USB. 


\section{Inicio}

Inicializar: Hardware, Variables, LCD

Habilitar interrupción TX -SCI para RECEPCIÓN

Protocolo de sincronización con SAICaP

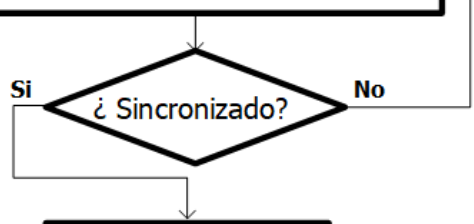

Recepción de datos

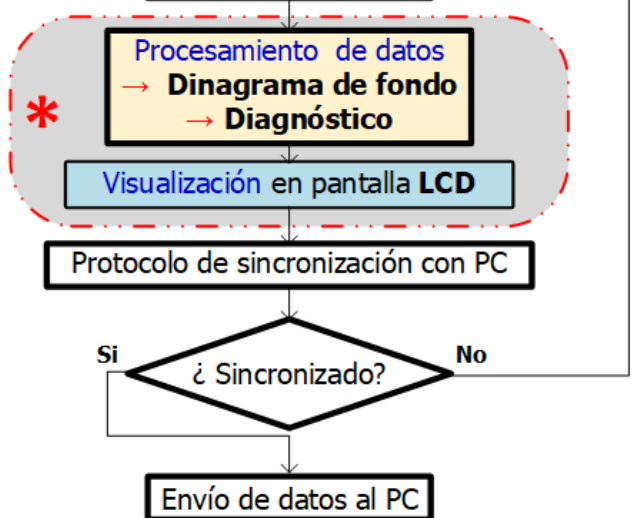

Fig. 12. Programa principal de SiRProl

El "núcleo o programa principal" (proceso dos) es el que se ejecuta cíclicamente, y aunque tiene control sobre los otros procesos, éstos una vez habilitados funcionan de manera independiente en una ejecución multitarea. La Figura 12 muestra el diagrama de flujo del proceso dos, el cual funciona de la siguiente manera:

a) Inicializa todos los módulos hardware, una vez realizada esta tarea, procede a realizar peticiones periódicas de datos al sistema SAICaP.

b) Evalúa si existen datos en el buffer de procesamiento de datos del SAICaP, si el buffer se encuentra vacío comienza con una rutina de espera habilitando una interrupción del módulo SCI, que permite evaluar si algún dato de sincronización es enviado como "petición de datos listos" desde el SAICaP.

c) Una vez el SAICaP ha enviado el dato de sincronización, y los dos sistemas se sincronizan, SAICaP realiza el volcamiento de los datos del buffer, a través del protocolo ZigBee; simultáneamente SiRProL entra en un estado de alerta, y por medio de la interrupción SCI se capturan todos los datos que envía de manera inalámbrica SAICaP, de tal manera que al final se tienen 700 datos en el SiRProL.

d) Estando residente ahora los datos en el SiRProL, se procede con el procesamiento de todos los datos y la visualización de resultados, obteniendo un diagnóstico y un dinagrama de fondo. En el siguiente numeral se presenta en detalle este componente del software, ver $(*)$ en Figura 12.

e) Teniendo ahora un diagnóstico y un dinagrama de fondo, SiRProL intenta sincronizarse por medio del puerto USB o vía Bluetooth, con un computador (PC), utilizando el mismo protocolo de sincronización desarrollado para enlazar el SiRProL y el SAICaP, la diferencia es que ahora el SiRProL es quien envía el carácter de sincronización y espera un carácter de vuelta, para enviar los datos al computador (PC).

f) Finalmente, el algoritmo comienza de nuevo, con la espera de nuevos datos del SAICaP para ser procesados.

\subsubsection{Procesamiento de datos, diagnóstico y visualización}

Este componente del proceso dos (* en Fig. 12), del software desarrollado para el SiRProL posee seis módulos (Fig. 13), los cuales se encargan de:

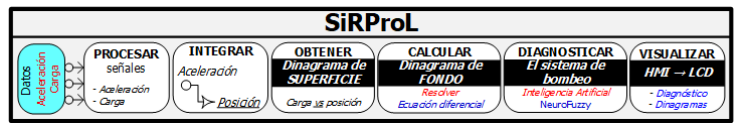

Fig. 13. Procesamiento y visualización.

i. PROCESAR señales (aceleración y carga) Los datos de carga y aceleración son almacenados por el SiRProL en vectores separados. Cada dato en el vector de aceleración corresponde en el mismo tiempo (t) con un dato de carga.

A la señal de aceleración, en un primer paso del procesamiento se le aplica un filtro pasa-bajos, obteniendo una nueva señal donde muchos de los componentes indeseados (ruido) han desaparecido. Para mejorar aún más esta señal, se le aplica un filtro digital no recursivo de promedio de muestras (non recursive moving average filter), el cual relaciona las muestras pasadas y futuras de un determinado punto en el tiempo discreto, es decir, 
realiza el promedio central de la señal tomando las muestras punto a punto. Estos dos filtros funcionan en cascada para tener una señal de aceleración a partir de la cual se puede obtener finalmente la señal de posición.

A la señal de carga, para acondicionarla, se le aplica también un filtro digital no recursivo de promedio de muestras

ii. INTEGRAR aceleración,

La señal de aceleración se integra numéricamente dos veces, para obtener la posición instantánea de la barra pulida, aplicando el procedimiento presentado en (Meneses et al., 2015).

iii. OBTENER el dinagrama de superficie.

Se correlacionan las señales procesadas de carga y posición para cada instante (t) de una carrera (stroke), y se obtiene el dinagrama de superficie.

iv. CALCULAR el dinagrama de fondo

Como ya fue expuesto, se requiere el dinagrama de fondo para diagnosticar el comportamiento de la bomba del sistema, la cual se encuentra a miles de metros de profundidad. El dinagrama de superficie, muestra: a) el valor numérico de la fuerza instantánea que actúa en la superficie sobre la barra pulida, y no la fuerza que actúa a miles de metros de profundidad sobre la bomba. b) El desplazamiento (posición) instantáneo de la barra pulida en la superficie, y no el desplazamiento real del pistón de la bomba.

Como se evidencia, lo medido en la superficie incorpora distorsiones que enmascaran el verdadero comportamiento de la bomba de fondo. Por lo tanto, en este proyecto para obtener el dinagrama de fondo, se utilizó: un modelo matemático, un método de cálculo y una implementación computacional.

El modelo matemático utilizado en este proyecto, se basó en el desarrollado por Pavlik (Pavlik, 1981), quien basó su desarrollo en el modelo de Gibbs (Gibbs y Nelly, 1966). Es aplicable solamente en pozos verticales pudiendo poseer una sarta de varillas combinada. El modelo incluye una formulación para el amortiguamiento derivado de la interacción entre la sarta de varillas, el fluido y la tubería de producción, y la posibilidad de utilizar sartas de varillas de fibra de vidrio. En el modelo está incorporada la inercia del líquido y el comportamiento visco-elástico del material de las varillas. El modelo asume un flujo newtoniano laminar a través del anular comprendido entre la sarta de varillas y la tubería de producción.

El modelo utilizado refleja el comportamiento dinámico de la interacción entre el crudo bombeado, la sarta de varillas y la tubería de producción, materializándose en una ecuación diferencial de segundo orden en derivadas parciales y sus correspondientes condiciones de frontera, denominada "Ecuación de Onda" (Fig. 14).

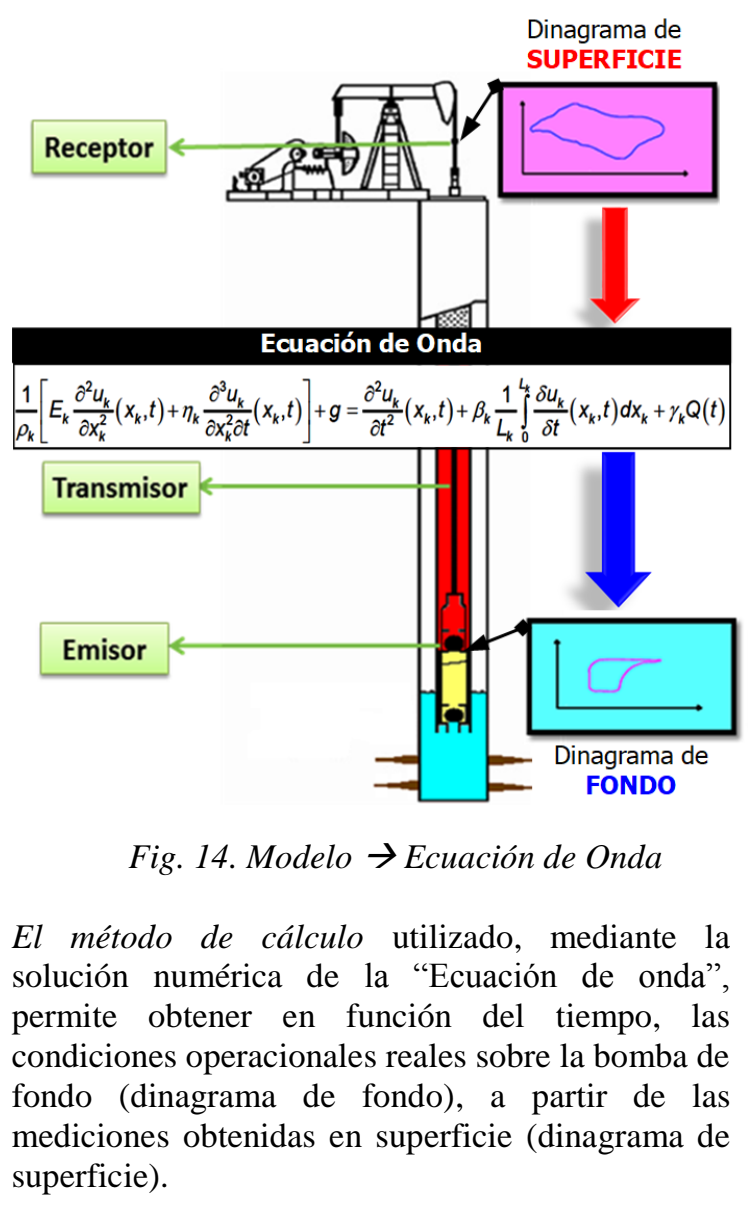

La implementación computacional (Fig. 15) fue desarrollada programando la solución numérica de la ecuación de onda, sobre el sistema embebido (SiRProl), obteniendo en tiempo real y de manera local, el dinagrama de fondo.

v. DIAGNOSTICAR el sistema de bombeo Eliminando la intervención de un experto humano, este módulo de software debe identificar con precisión y rapidez los problemas en el pozo, reconociendo de manera automática, los patrones de falla del sistema de bombeo mecánico, a partir de los dinagramas de fondo, anticipando así los problemas a través de su pronta identificación. 
En proyectos paralelos se desarrollaron estrategias que utilizando una arquitectura Neuro-fuzzy (Caballero y Salamanca, 2011; Garavito y Meneses, 2014) permiten obtener un modelo difuso (o modelo fuzzy), que diagnostica a partir de los dinagramas de fondo, las fallas presentes en un sistema de bombeo mecánico. El modelo fuzzy obtenido no puede ser llevado directamente a un sistema embebido, por lo que en otro proyecto (Zarate, 2016) se desarrolló una aplicación software, que a partir de un modelo fuzzy, genera el código para un sistema embebido especifico, que en este caso corresponde a la plataforma de desarrollo embebida Freescale TWR-K70F120M.

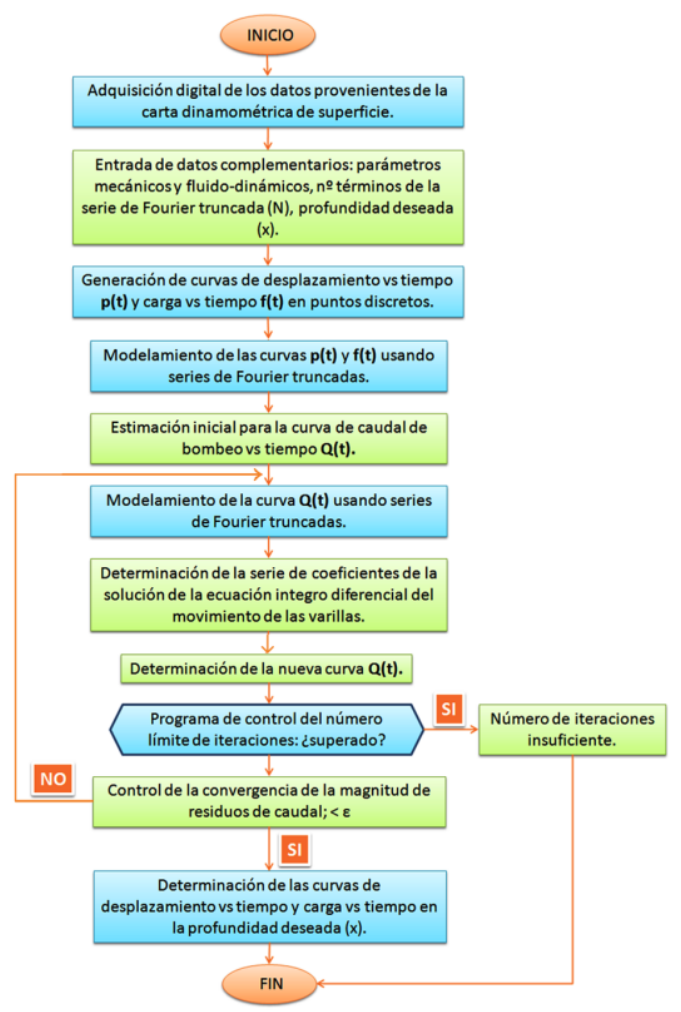

Fig. 15. Ecuación de Onda $\rightarrow$ Diagrama de flujo de la implementación computacional

vi. VISUALIZAR el diagnóstico y los dinagramas Finalmente, se despliega (visualiza) el dinagrama de fondo obtenido y el diagnóstico del sistema, través del HMI del SiRProL (pantalla LCD), lo que permite al operador del pozo visualizar de manera inmediata, el comportamiento del sistema de bombeo mecánico y transmitir (diagnóstico y dinagramas) de ser requerido, a una estación central de recolección de información.

\subsection{Comunicación inalámbrica}

\section{Universidad de Pamplona}

$$
\text { I. I. D. T. A. }
$$

El módulo XBEE PRO de Digi, utiliza el protocolo ZigBee, el cual a su vez utiliza como medio físico el estándar IEEE 802.15.4. El proceso de autenticación de la integridad de los datos, y la redundancia, ya están montados sobre el protocolo, sin embargo, se puede configurar el número de intentos de comunicación entre los puntos si un dato no se recibe debidamente. En este proyecto se adicionó al protocolo una sincronización entre los sistemas SAICaP y SiRProL para garantizar la comunicación entre ellos (Fig. 16).

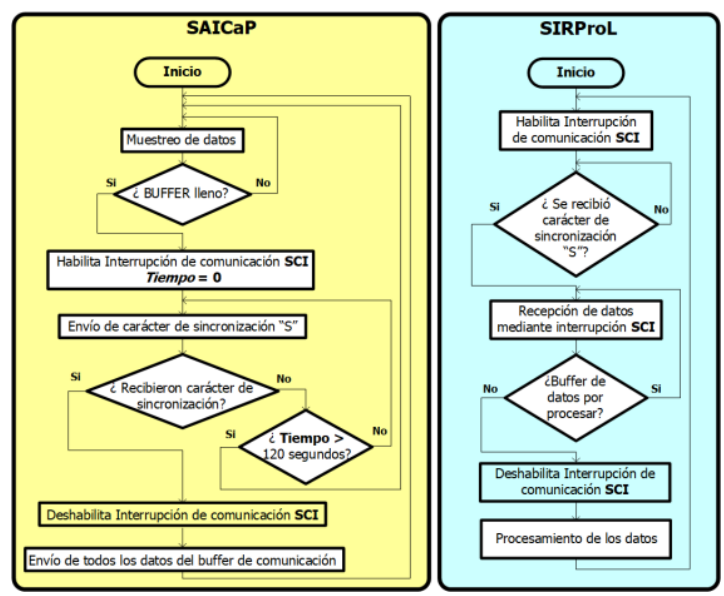

Fig. 16. Protocolo de comunicación

Los datos son empaquetados (Fig. 17) en caracteres ASCII para ser enviados. Si el vector de aceleración contiene un dato numérico e.g -1234, y el dato de carga correspondiente es e,g. +891234, la trama se convierte en caracteres ASCII de 8 bits, con lo cual cada número es representado por un binario que corresponde con su carácter en ASCII.

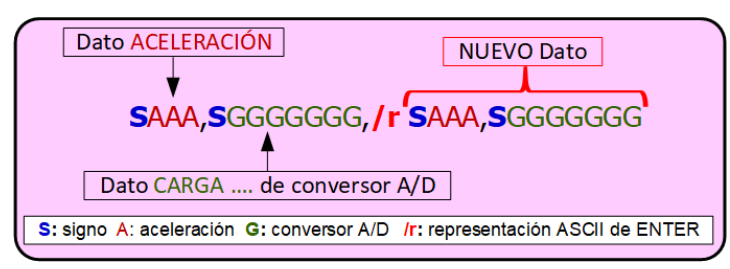

Fig. 17. Empaquetamiento de los datos

El envío del carácter de fin de línea y retorno de carro en ASCII, facilita la identificación de los datos correspondientes entre sí para aceleración y carga y, además permite una mayor organización estructural del programa. Para la sincronización de los sistemas, una vez el buffer de datos se ha llenado, SAICaP envía un carácter de sincronización que es "S" seguido del comando /r, 
al mismo tiempo que esto sucede y por medio de la interrupción de recepción, espera a que el SIRProL envié el carácter de respuesta "Y", esto durante 120 segundos y de igual manera emitiendo periódicamente el carácter " $\mathrm{S}$ " debido a que no sabe si el SIRProL tiene activa la recepción inalámbrica. Teniendo en cuenta que el protocolo Zig-Bee montado sobre el módulo XBEE PRO permite una comunicación Duplex, en el evento de que el SAICaP se encuentra emitiendo al mismo tiempo que el SIRProL emite la respuesta, no se tendría ningún inconveniente. Si durante los 120 segundos de sincronización, el SAICaP no recibe algún carácter de parte del SIRProL, procederá a un nuevo muestreo de datos. Este caso se puede dar debido a que el muestreo tarda aproximadamente 50 segundos, la transmisión aproximadamente un minuto, y el procesamiento puede llegar a los dos minutos, de esta manera el SAICaP estará dispuesto a transmitir dos o tres paquetes de datos, mientras espera por la petición del SIRProL. En el SIRProL la comunicación estará abierta a la espera de nuevos datos, una vez se hayan terminado de procesar los datos pasados.

\section{RESULTADOS}

La arquitectura diseñada y aquí presentada, permitió el desarrollo del SmartOMP (Smart Oil Mechanical Pumping), prototipo de un producto industrial de naturaleza mecatrónica, para la obtención automática del dinagrama de fondo de pozo y el diagnóstico en tiempo real del sistema de bombeo. Concebido para su instalación en el pozo 74 de CEC, es aplicable a cualquier pozo que utilice el bombeo mecánico como sistema de levantamiento artificial

\section{CONCLUSIONES}

¿Es posible diseñar y construir una solución propia e innovadora de hardware y software, que establezca las bases para el concepto propio de "pozo y campo inteligente"? El desarrollo del SmartOMP evidencia que, si es posible desarrollar en el contexto nacional, productos de naturaleza mecatrónica dirigidos a la industria petrolera, que contribuyan al desarrollo nacional aumentando la producción de los campos petroleros colombianos.

\section{RECONOCIMIENTO}

Este trabajo hizo parte del proyecto de investigación 8556 "Desarrollo de un prototipo de pozo inteligente para Campo Escuela Colorado", financiado por la Vicerrectoría de Investigación y Extensión de la UIS (Universidad Industrial de Santander).

\section{REFERENCIAS}

Al-Mubarak S. (2008). Real-time Reservoir Management from Data Acquisition through Implementation: Closed-Loop Approach, Intelligent Energy Conference and Exhibition, Amsterdam (The Netherlands), 111717-MS SPE Conference Paper.

Araque, JA, Rodríguez, JLD, Guerrero A. (2017) Optimización por recocido simulado de un convertidor multinivel monofásico con modulación PWM sinusoidal de múltiple portadora. Revista Tecnologías de Avanzada, ISSN: 1692-7257.

Bermeo, WL, Jr, AB de Souza, Fernández T, Honorio D, Nogueira dos Reis L, Barreto L. (2017). Control modo deslizante aplicado en la malla de corriente para una aplicación de una base-DSP para el control de posición de un motor de inducción de jaula de ardilla. Revista Tecnologías de Avanzada, ISSN: 1692-7257

Caballero F. y Salamanca J. (2011). Herramienta de aplicación software para clasificación de patrones de datos implementando una arquitectura Neuro-fuzzy, Universidad Industrial de Santander (UIS), Bucaramanga (Colombia). Tesis de pregrado.

de Best L. (2012). Shell's Smart Fields Sustaining and Accelerating Benefits from Intelligent Fields, Intelligent Energy International, Utrecht, 150407-MS SPE Conference Paper.

de Best L. (2006). Smart Fields - Making the Most of Our Assets, SPE Russian Oil and Gas Technical Conference, Moscow (Russia), 103575-MS SPE Conference Paper.

Dickinson R. y Jennings J. (1990). Use of patternrecognition techniques in analyzing downhole dynamometer cards, SPE Production Engineering, Vol. 5, No. 2, SPE-17313-PA.

Gabor T. (2003). Sucker-Rod Pumping Manual, Editor PennWell Books, ISBN 0-87814-899-2 PennWell Books, USA.

Gao H. (2007). A Literature Review on Smart Well Technology, Production and Operations Symposium, Oklahoma (USA.), 106011-MS SPE Conference Paper. 
Garavito F. y Meneses E. (2014). Sistema Neurofuzzy: prospectiva de aplicación en la detección de fallas en equipos de subsuelo de unidades de levantamiento mecánico, Universidad Industrial de Santander (UIS), Bucaramanga (Colombia). Tesis de pregrado.

Gibbs, S. y Nelly, A. (1966). Computer diagnosis of down hole conditions in sucker rod pumping wells, Journal of Petroleum Technology, Vol. 18, No.1, SPE1165PA.

Gómez A. E., Archila J.F., Meneses, J.E. (2013). Adquisición y tratamiento de señales de un acelerómetro triaxial MEMS, para la medición del desplazamiento de una extremidad inferior, Revista Colombiana de Tecnologías de Avanzada Vol. 1, No. 21, pp. 113-118.

McCoy J. y Podio L. (1995). Method and Apparatus for measuring pumping rod position and other aspects of a pumping system by use of an accelerometer, Patent US5406482.

Meneses J.E., García J.D. y Ferreira D.A. (2015). Acelerómetros MEMS en el desarrollo de pozos y campos petroleros inteligentes, Revista Colombiana de Tecnologías de Avanzada Vol. 2, No. 26, pp. 128-135.

Meneses J.E., Niño J.D., y García F.A. (2017). KINE-UIS: modelamiento de video para la adquisición de la velocidad y aceleración angular instantánea de un sólido rígido, Revista Colombiana de Tecnologías de Avanzada Vol. 2, No. 30.

Moises G., Rolim T. y Formigli J. (2008). GeDIg: Petrobras Corporate Program for Digital Integrated Field Management, Intelligent Energy Conference and Exhibition, Amsterdam, 112153-MS SPE Conference Paper.

Pari M., N. y Kabir A., H. (2009). Smart wellBenefits, Types of Sensors, Challenges, Economic Consideration, and Application in Fractured Reservoir, SPE Saudia Arabia Section Technical Symposium, 126093-MS SPE Conference Paper.

Pavlik N. (1981). Schwingungsmodelle fuer die gestage bewegung in forderden erdol sandin und ihre Anwendug auf die analyse von dyn diagramment, Clausthal (Alemania), Universidad Técnica de Clausthal. Tesis de Doctorado.

Sanchez J. P., Festini D. y Bel O. (2007). Beam Pumping System Optimization Through Automation, Latin American \& Caribbean Petroleum Engineering Conference, Buenos Aires (Argentina), SPE 108112.
Sletcha B., Vivas, C., K. Saleh, F., Ghalambor A. y Salehi S. (2020). Digital Oilfield: Review of Real-time Data-flow Architecture for Upstream Oil and Gas Rigs, International Conference and Exhibition on Formation Damage Control, Lafayette, (Louisiana, USA), 199298-MS SPE Conference Paper.

Zarate C. (2016). Preprocesador de fuzzy inference system (FIS) y motor de inferencia difusa para la plataforma de desarrollo embebida Freescale TWR-K70F120M. Universidad Industrial de Santander (UIS), Bucaramanga (Colombia). Tesis de pregrado. 